\title{
모델기반 예측적고장결정 기법(AFD) 적용 연구 A Study on the Application of Model Based Anticipatory Failure Determination (AFD)
}

\author{
이철우 ${ }^{* 11}$, 구기홍 ${ }^{1)}$, 김대용 ${ }^{1)}$, 손명존), 이중윤 ${ }^{3)}$ \\ Cheolwoo Lee ${ }^{* 11}$. Kihong $\mathrm{Ku}^{1)}$, Daeyong Kim ${ }^{1)}$ Joonyun Lee ${ }^{2)}$ MyeongJo Son ${ }^{3)}$
}

$$
\text { [ 초 록 ] }
$$

종래 리스크 예측/식별 기법들이 과거 경험한 유사 사례가 없는 미지의 미래 체계에 리스크 요소를 예측/식별하는데는 많은 제한 이 된다. 특히 실전 경험이 없는 미지의 미래 무인무기체계는 운용개념에 대해 예측하기가 매우 어려운데다가 유인에 의한 임기응변 조차 제한되어 무인무기체계의 실전 배치시 그 임무 달성에 실패하거나 예기치 못한 사건/사고를 발생시킨 사례가 적지 않다. 이를 극복하기 위해 예측적 고장결정 기법을 모델기반 시스템 공학(MBSE)과 결합하여 최근 군에서 미래 무인 무기체계 개발사업의 일환 으로 추진 중인 국방 무인OO차량개발사업의 리스크 해소방안에 적용한 사례를 소개하여 미래 무인무기체계 개발사업의 리스크 해 소와 성공 가능성을 높이는데 기여하고자 하였다.

\section{[ ABSTRACT ]}

Conventional risk prediction/identification techniques have many limitations in predicting/identifying risk factors in an unknown future system without similar cases experienced in the past. In particular, an unknown future unmanned weapon system with no practical experience is very difficult to predict about its operational concept, and even ad hoc response by manned is limited. There are not a few cases. In order to overcome this, we introduced a case where the predictive failure determination technique was combined with model-based system engineering (MBSE) and applied to the risk reduction plan of the defense unmanned $O O$ vehicle development project, which is currently being promoted by the military as part of the future unmanned weapon system development project It aimed to contribute to solving the risk and increasing the possibility of success in the future unmanned weapon system development project.

Key Words : Risk Identification (위험 식별), Anticipatory Failure Determination (AFD, 예측적 고장결정), 모델기반 시스템공 학(MBSE, Model Based Systems Engineering),

\section{1. 서 론}

종래 리스크 예측/식별 및 관리에 사용되는 주요 기법들로 는 FMEA, ETA, FTA 등의 리스크 관리기법들이 있다 하지 만 이들 기법들은 기존에 경험한 사고나 고장 사례가 많을 경 우 리스크 예측/식별에 유용하나 유사한 시스템에 대한 경험 이 없거나 부족한 신규 시스템 영역에 대한 리스크 예측/식별 이나 관리에는 적용이 제한된다. 게다가 불쾌하고 부정적인 생각을 '거부'하려는 인간의 심리적 경향으로 인해 기존의 리

1)현대로템(Hyundai Rotem), 2)SETechnology, 3)포항공대 엔지니어링 대학원(Graduate School of Engineeering, Pohang University of Science and Technology, Korea)

* Corresponding author, E-mail: syslee@hyundai-rotem.co.kr Copyright (c) The Korean Institute of Defense Technology

Received: November 27, 2020

Accepted: December 11, 2020
스크 예측/식별기법들이 보다 적극적으로 미래 리스크 요소를 예측하고 식별하는데 많은 어려움이 있다. 그 결과 미지의 신 규 시스템의 개발이나 운용 사업은 예측/식별치 못한 심각한 리스크에 노출될 수 있다.

특히 실전 경험이 없는 미지의 미래 무기체계는 운용개념에 대해 예측하기가 매우 어려우며 그로 인하여 리스크에 대해 선제적으로 식별과 대응에 실패하여 무기체계의 실전 배치시 무용지물이 된 사례가 적지 않다. 이에 본 논문 2장에서는 신 규 무기시스템에 예측치 못한 리스크의 사전 식별과 대처에 상대적으로 유용한 예측적 고장결정 (이하 $\mathrm{AFD}$, Anticipatory Failure Determination) 기법을 소개하면서 부정적 리스크에 대한 인간 심리의 원천적인 거부 성향을 조명하고 리스크 예 측/식별에 대한 발상의 전환, 그리고 이를 미지의 리스크 식별 방법론으로 발전시킨 6단계를 개략 소개하였다. 이어서 3장에 서는 예측적 고장결정(이하 $\mathrm{AFD})$ 기법의 6단계 절차와 방법을 
시스템 공학분야에서 오래전부터 일반화된 모델기반 시스템 공학(MBSE, Model Based Systems Engineering)과 결합하 여 $\mathrm{AFD}$ 의 예지적 측면을 한층 발전시킨 방법론을 제시하였다. 그리고 4장에서는 이를 최근 미래 무인무기체계 개발사업의 일환으로 추진중인 국방 무인OO차량개발사업의 리스크 해소 방안에 적용한 사례를 소개하여 미래 무인무기체계 개발사업 의 리스크 해소와 성공 가능성을 높이는데 기여하고자 하였다.

\section{2. $\mathrm{AFD}$ 기법의 리스크에 대한 발상의 전환}

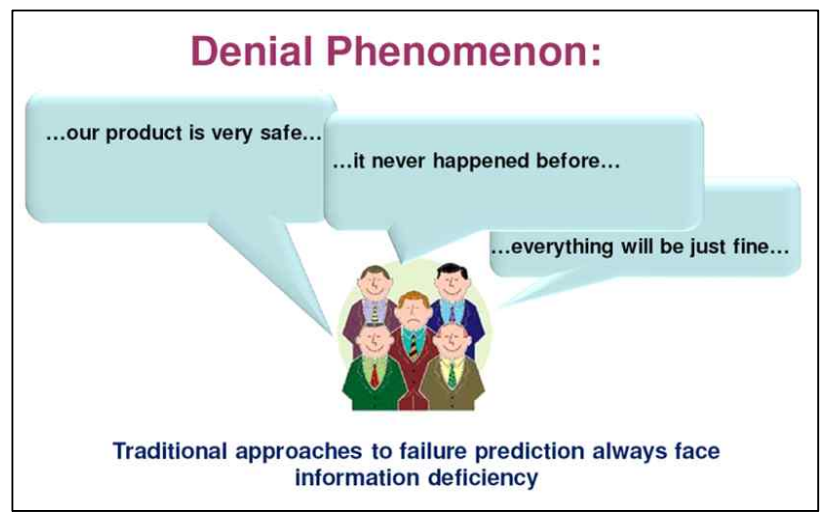

그림 1. 기존 리스크 예측/식별 기법의 심리적 저해요인

Fig. 1. Psychological impediments of existing risk prediction/identification techniques

인간의 본성에는 리스크나 사고처럼 불쾌한 일이나 사건에 대하여 생각하기를 '거부'하거나 '소극적'으로 접근하려는 인간 의 심리적 경향이 내재되어 있다. 이에 구 소련(현 러시아)를 중심으로 “시스템과 관련된 리스크가 발생하기 전에 이를 사 전 확인하는 시스템적인 접근법”[1]으로 정의되는 예측적고장결 정(AFD)방법론이 발달하였는데 이는 불쾌하고 부정적인 생각 을 '거부'하는 인간의 심리적 경향으로 인해 기존 리스크 예측 기법들에 내재된 심리적 제한 요소를 TRITZ의 창의적인 방법 론으로 극복한 접근법이다. ${ }^{[2]}$

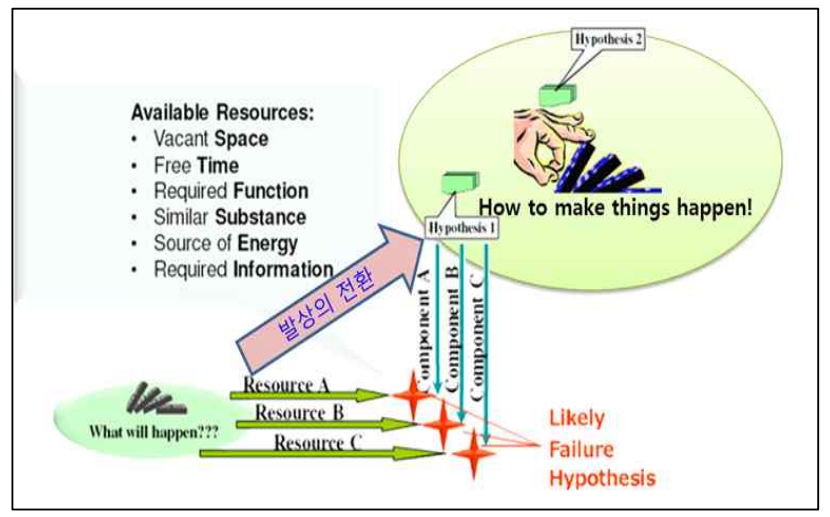

그림 2. $\mathrm{AFD}$ 의 발상의 전환

Fig. 2. Change of the idea of AFD

즉, 기존에 리스크 예측 기법들에서는 "어떤 나쁜 일이 발생 할 것인가?", "어떻게 하면 이 시스템의 사고/침탈/고장을 방 지할 것인가?”라고 하는 부정적 예측과 사고를 요구하는 반면
에 $\mathrm{AFD}$ 의 접근 방법에서 리스크 예측 참여자들은 공격자의 입장에 서서 "어떻게 하면 이 시스템을 파괴, 공격,와해 혹은 고장낼 것인가?”라는 공격적이고 적극적인 발상의 전환을 시 도한다. ${ }^{[1]}$ 이에 따라 참여자들은 심리적 제한없이 기존에 방법 에서 예측하지 못했던 각종 기발한 고장이나 사고 사례를 창 발적으로 도출 할 수 있다. 그리고 $\mathrm{AFD}$ 의 접근 방법에서는 이렇게 도출된 고장이나 사고발생이 예상되는 시스템의 관련 자원에 대해 식별하고 이에 대한 대응책을 마련하게 된다.

이러한 $\mathrm{AFD}$ 의 발상 전환은 그림 3 의 좌측에 $\mathrm{AFD}$ 절차와 같이 문제정의 $\rightarrow$ 정상시나리오 확인 $\rightarrow$ 문제상황 역전개 $\rightarrow$ 시스템 고장 발생야기 $\rightarrow$ 고장시스템 자원식별 $\rightarrow$ 새로운 해결책 도출 절차로 구체화된다. 그중에 특히 공격적인 발상법으로 문제상황 역전 개(3단계 절차)와 시스템 고장발생야기(4단계 절차) 활동을 통 해 $\mathrm{AFD}$ 의 발상을 전환한 사고 방식을 적용하여 대상 시스템 이나 그 운용에 대해 창의적인 파괴, 공격, 와해 혹은 고장유 도 방법을 찾게 되며 고장 시나리오별로 필수 관련 자원을 식 별(5단계)하고 이에 대한 대응책을 마련하게 된다(6단계)

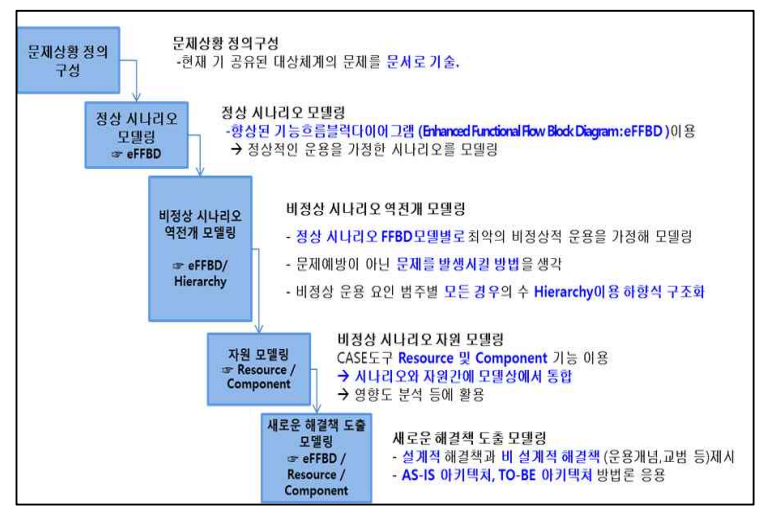

그림 3. $\mathrm{AFD}$ 6단계 절차

Fig. 3. AFD 6 step procedure

\section{3. $\mathrm{MBSE}$ 를 통한 $\mathrm{AFD}$ 기법의 체계적 적용}

이러한 AFD 발상 전환, 절차 및 방법은 기존 시스템 공학 분야에서 널리 적용중인 모델기반 시스템공학(이하 MBSE)방법 론과 융합할 경우 매우 효과적일 수 있다. 즉 $\mathrm{AFD}$ 의 정상시 나리오 작성(2단계)를 $\mathrm{MBSE}$ 거동모델 $\mathrm{eFFBD}$ 등을 이용하여

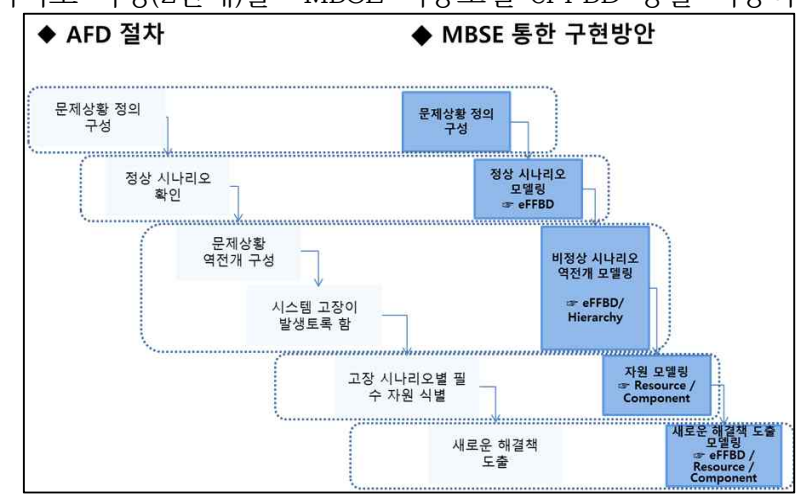

그림 4. MBSE를 통한 $\mathrm{AFD}$ 기법의 구현

Fig. 4 Implementation of AFD technique through MBSE 
객관화 및 가시화 모델링하고 이를 바탕으로 문제상황 역전개 (3단계)와 시스템 고장발생야기(4단계) $\mathrm{AFD}$ 리스크 예측/식별 /관리에 활용할 수 있으며 또한 결함 시나리오와 관련된 자원 모델링에는 MBSE 구성품모델과 자원 모델을 활용할 수 있 다.(5단계) 또한 단일한 MBSE 도구를 활용하여 상기 기술한 기능 , 거동, 컴포넌트 그리고 자원 모델들을 통합하여 리스크 를 모델링하고 관리할 수 있다.(그림4 참조) ${ }^{[3],[4]}$

\section{4. 미래 무인OO시스템 개발사업 리스크 예측/식별 적용사례}

실전 경험이 없는 미지의 미래 무기체계는 운용개념에 대해 예측하기가 매우 어려우며 그로 인하여 리스크에 대해 선제적 으로 예측/식별과 대응에 실패하여 무기체계의 실전 배치시 무용지물이 된 사례가 적지 않다. 특히나 예측/식별하지 못한 상황에 사람의 임기응변으로 대응이 가능한 유인체계와 달리 무인체계의 경우에는 사람의 임기응변이 제한되거나 불가능한 경우가 많아 전력화시 야전이나 운용환경에서 실패나 사고로 귀결된 사업을 많이 찾을 수 있다. (2차대전기 독일군의 골리 앗 무인전차, 한국 소방로봇사업, 테슬라 자율주행차량 사망사 고 등) ${ }^{[5]}$

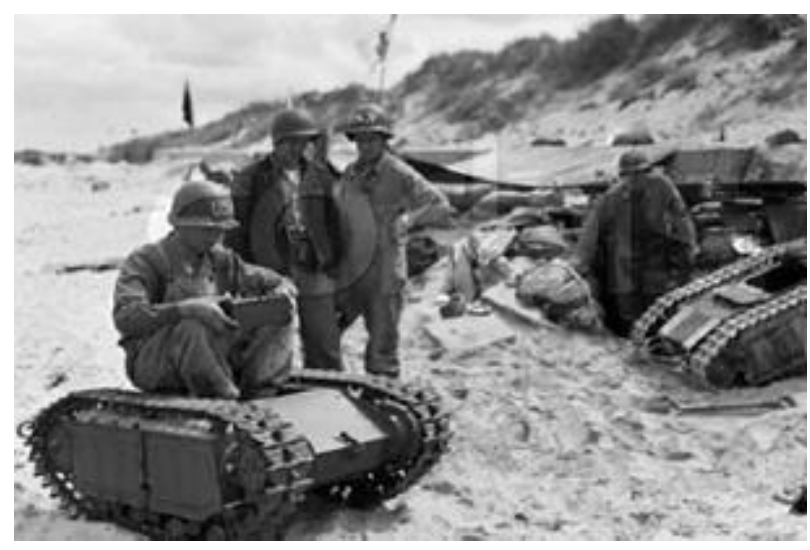

그림 5. 2차대전기 연합군에 포획된 독일군의 골리앗 무인전차

Fig. 5. German Goliath unmanned tank captured by the Allied Forces of World War II

이에 본 연구에서는 군의 미래 무인OO시스템 개발사업의 예상치 못한 리스크 발생을 최소화 하기 위하여 리스크 예측/ 식별에 2장과 3장에서 소개한 $\mathrm{AFD}$ 의 발상법과 이를 MBSE에 응용한 분석 방법을 적용하였다. 그 결과 그림 6 에서와 같이 미래 무인OO시스템 개발사업의 관련 이해관계자들은 비록 미 래 무인OO시스템과 운용에 대한 경험이 부족함에도 불구하고 종래 리스크 예측/식별/관리기법에서 예측/식별이 제한되었던 미래 무인 $\mathrm{OO}$ 시스템에 대하여 MBSE기법을 활용하여 예상되 는 운용 시나리오 초안을 모델링하고 집단 지성을 이용하여 미지의 운용 시나리오를 보완 및 상세화 할 수 있었다.

그리고 이를 토대로 $\mathrm{OO}$ 작전운용시나리오에 대해 공격자의 입장에서 상상할 수 있는 각종 파괴, 공격, 와해 혹은 고장야 기 상황을 보다 적극적이고 창의적으로 상상하고 도출할 수

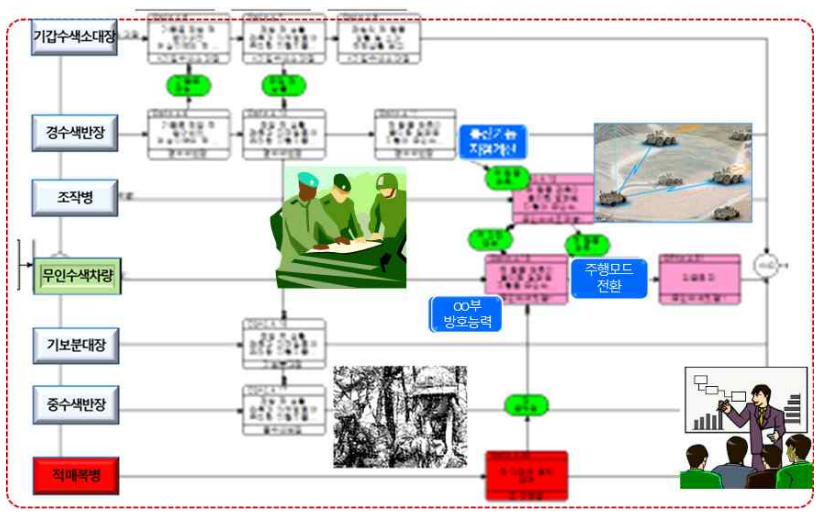

그림 6. 문제상황 역전개 통한 고장발생 시나리오 분석

Fig. 6. Analysis of failure occurrence scenarios through reversal of problem situations

있었고 (그림 6 의 붉은색 및 분홍색 부분) 도출된 고장, 위해 예측항목별로 대응책을 모델링하였다. (그림 6의 청색 부분) 이러한 대응책 모델링을 종합하여 대응책 관련 요구사항(OR) 를 수립하고 관련 구성품을 식별하여 구성품 설계에 반영하였 다.(그림7 부분) ${ }^{[6]}$

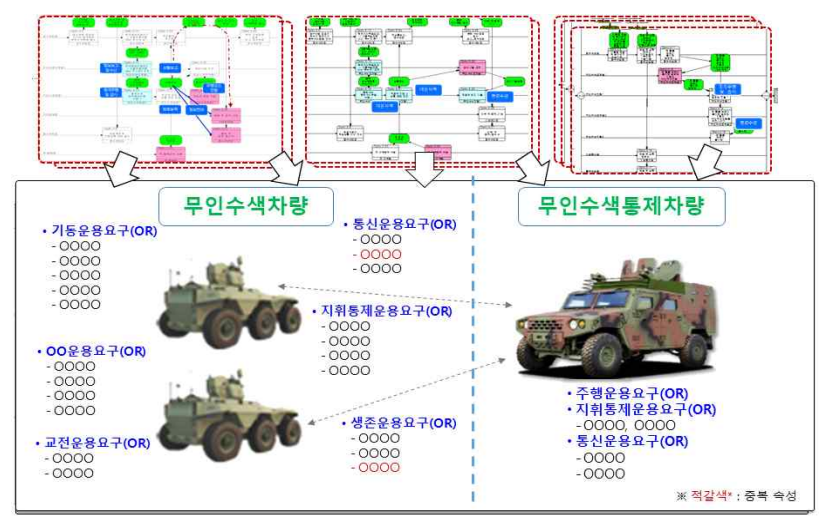

그림 7. 대응책 운용요구(OR) 수립 및 관련 구성품 식별

Fig. 7. Establishment of countermeasure operation request $(\mathrm{OR})$ and identification of related components

\section{5. 결 론}

기존 FMEA 등 리스크 예측/식별 및 관리 기법들로는 유사 한 시스템 개발이나 운용 경험이 부족한 신규 영역에 대한 리 스크 예측/식별과 대처에 제한되는 상황에서 이를 극복하는 대안으로 $\mathrm{MBSE}$ 기반으로 $\mathrm{AFD}$ 를 적용할 경우 미래 무기시스 템에 경험이 부족함에도 불구하고 각종 고장야기 상황을 보다 적극적이고 창의적으로 예측/식별하고 사전에 대처할 수 있음 을 군의 미래 무인OO시스템 개발사업에 적용을 통해 확인하 였다. 특히 미래 무인OO시스템과 운용에 대한 경험이 부족함 에도 불구하고 미래 무인OO시스템 개발사업의 관련 이해관계 자들은 MBSE기법을 활용하여 예상되는 운용 시나리오 초안을 모델링함으로써 집단 지성을 이용하여 미지의 운용 시나리오 를 지속적으로 보완 및 상세화 할 수 있었고 대응책을 수립할 
수 있었다. 향후 미지의 무기체계 특히 미래 무인무기체계에 대한 리스크 예측/식별/관리에 $\mathrm{MBSE}$ 기반 $\mathrm{AFD}$ 의 발상과 방 법을 확대 적용하여 사업 성공에 기여할 수 있기를 기대한다.

\section{References}

[1] Avner Engel, "VERIFICATION, VALIDATION, AND TESTING OF ENGINEERED SYSTEMS”, A JOHN WILEY \& SONS, INC., PUBLICATION, U.S.A, 2010

[2] Stan Kaplan, "Svetlana Visnepolschi, Boris Zlotin and Alla Zusman, New Tools for Failure and Risk Analysis", Ideation International Inc. .2005

[3] Lee Cheol Woo, "Application of AFD and CASE Analysis for the Safety Improvement of the Ground Combat Vehicle during River Crossing Operation", Ajou University Graduate School for Systems Engineering, Suwon, 2015.

[4] Vitech, "CORE User Guide v9.0”, USA, 2017,

[5] Lee Cheol Woo, Koo, Ki Hong, Lee Joong Yoon, Lim Gwan Taek, Son Myeong Jo, "A Study on Managing the Uncertainty of Operational Concept of an Unknown Future Unmanned System", Korea Institute of Military Science and Technology, Annual Conference Proceedings, 2018,

[6] Lee Cheol Woo, Son Myeong Jo, "Final Report of the Architecture Development and Analysis of Autonomous OO Vehicle". Hyundai Rotem, Uiwang-si, 2017. 